\title{
Suppression of Self-Excited Vibrations in Rotating Machinery Utilizing Leaf Springs
}

\author{
Chang Wang1 - Jun Liu'1,2,* - Zhiwei Luo ${ }^{3}$ \\ 1Tianjin Key Laboratory for Advanced Mechatronic System Design and Intelligent Control, \\ Tianjin University of Technology, China \\ 2National Demonstration Centre for Experimental Mechanical and Electrical Engineering Education, \\ Tianjin University of Technology, China \\ 3Organization of Advanced Science and Technology, Kobe University, Japan
}

When rotating machinery is operated above the major critical speed, self-excited vibrations appear due to internal friction of the shaft. Internal frictions are classified into hysteretic damping due to the friction in the shaft material and structural damping due to the dry friction between the shaft and the mounted elements. In this paper, a method to suppress the self-excited vibration using leaf springs are proposed. The structural damping is considered as the internal damping. The characteristics of a rotor with leaf springs are investigated systematically by using simulative and theoretical analyses. The validity of the proposed method is also proved by experiments.

Keywords: self-excited vibration, internal damping, vibration suppression, leaf spring, experiment

Highlights

- A rotor system with internal frictions and leaf springs is analysed by using numerical simulations and theoretical analyses systematically, and self-excited vibrations occur above the major critical speed.

- $\quad$ The self-excited vibrations can be suppressed by using leaf springs on the rotor system with internal frictions.

- The effectiveness of the proposed suppressing method is verified by experiments, simulations, and theoretical analyses.

\section{O INTRODUCTION}

In rotor systems, the friction created between a rotational part and a stationary part, such as a rotating disk and the surrounding air, is called external friction, and another friction that works within two rotating parts is called internal friction. In addition, the internal friction is further classified into hysteretic damping, which works in the inside part of the shaft material, and structural damping, which occurs due to the sliding between the shaft and mounted elements such as bearings and gears. It is well known that the self-excited vibration occurs above the major critical speed when the internal friction works in the rotor system [1]. Regarding the self-excited vibration caused by internal friction, many scholars have conducted extensive research. Queiroz [2] studied flow-induced instabilities known as "whirl" and "whip" on the lightly-loaded shaft supported by fluidfilm bearings and analysed self-excited vibrations. Bonello and Pham [3] presented a generic technique for the transient nonlinear dynamic analysis (TNDA) and the static equilibrium stability analysis (SESA) of a turbomachine running on foil air bearing (FABs). Their research revealed stabilities and self-excited vibrations of the rotor system. Boyaci et al. [4] carried out an investigation of the stability and bifurcation phenomena of the rotor-bearing system and found self-excited vibrations with very high amplitudes. Bykov and Tovstik [5] studied synchronous whirling and asynchronous self-excited vibrations on the statically imbalance rotor under action conditions of external and internal damping forces. Because the nonlinear characteristics of the internal friction are very complicated, obtaining succinct expression of the theory is very difficult. The mechanism of selfexcited vibrations caused by the internal friction was understood [1] to [5]. Ishida and Yamamoto [6] investigated characteristics of the subharmonic resonance of the order of $1 / 2$ on the rotor-bearing system with a nonlinear spring-restoring force and an internal damping force. The phenomena were understood that self-excited vibrations occurred under the forced autonomous system. In addition, many researchers studied self-excited vibrations due to other causes on the rotor system. Coudeyras et al. [7] presented a novel nonlinear method called the Constrained Harmonic Balance Method (CMBM), which is applied to solve the specific problem of disc brake squeal with extensive parameters and to predict self-excited vibrations. Han et al. focused on the experimental study for the dynamic characteristics of a permanent magnet (PM) disk-type motor rotor supported by an aerostatic gas bearing and analysed low-frequency vibrations caused by self-excited gas films [8]. Vlajic et al. [9] studied dynamic 
characteristics of a modified Jeffcott rotor with the torsional deformation and the rotor-stator contact and investigated self-excited backward whirling motions with the continuous stator contact. Hua et al. [10] presented the basic excitation mechanism and vibration characteristics on the coupled bending and torsional nonlinear dynamic model of a rotor system with a nonlinear friction, and the results revealed multiform complex nonlinear dynamic responses of the rotor system under rubbing. Nishimura et al. [11] explained self-excited vibrations in the vertical pump with a journal bearing and demonstrated the nonlinear steady-state vibration analysis of self-excited vibrations. Tadokoro et al. [12] focused on self-excited vibrations induced by the velocity-weakening friction in rotary contact systems. Chouchane and Amamou [13] analysed the bifurcation of the steady-state equilibrium point of the journal centre and predicted stable or unstable limit cycles from the equilibrium point at the major critical speed. Peletan et al. [14] proposed a quasi-periodic harmonic balance method (HBM) to deal with self-excited vibrations of the steady-state dynamic behaviour of rotor-stator contact problems.

It is well known that harmonic resonances can be avoided by controlling rotational speeds. However, since the self-excited vibrations occur within a wide range of rotational speeds, it is difficult to escape the occurrence of self-excited vibrations. In addition, the amplitude of vibrations increases with exponential growth if there is no limit cycle. Based on the two characteristics mentioned above, it is concluded that self-excited vibrations are more dangerous than the harmonic resonance. Some methods of suppressing self-excited vibrations have been proposed. Kligerman et al. [15] investigated the nonlinear behaviour of shaft supports at the boundaries and stability of a rotating system with an electromagnetic noncontact damper, and a closed-form solution for the radius of the limit cycle and the frequency of self-excited vibrations are obtained. Inoue et al. [16] researched the occurrence region and vibration characteristics of self-excited vibrations caused by the ball balancer. The results are also validated experimentally. However, the theoretical analyses of self-excited vibrations have been less commonly proposed.

In this paper, based on self-excited vibrations by causing the structural damping, a suppressing method by using leaf springs is proposed. The vibration characteristics of a rotor with leaf springs are systematically investigated using theoretical analyses and numerical simulations. The validity of proposed method is also verified by experiments.

\section{THEORETICAL MODEL}

\subsection{Dynamic Equations}

The theoretical model of the rotor system with leaf springs is shown in Fig. 1. The two degrees of freedom (2DOF) inclination model with the gyroscopic moment is adopted, and a rigid disk is mounted at the centre of a massless elastic shaft. To suppress self-excited vibrations due to the internal damping, a bearing is fitted to the shaft, and four groups of leaf springs are placed to contact with the outer race of the bearing in four directions shown in Fig. 1. The rectangular coordinate system $O-x y z$ is established, and the $z$-axis coincides with the bearing centreline. The point $O$ is the geometrical centre of the disk. The line $O A$ is the centreline of the disk, and the line $O B$ is the tangent of the shaft at Point $O . \tau$ is the angle between lines $O A$ and $O B$, which represents the imbalance of the rotor system. $\theta$ is the angle between lines $O z$ and $O B$, and it is the inclination angle of the shaft at the position of the disk. $\theta_{x}$ and $\theta_{y}$ are projections of $\theta$ on planes $x z$ and $y z$, respectively.

It is assumed that the internal damping caused by the sliding between the disk and the shaft works in this system. In addition, the sliding here means that the inclined elastic rotor whirls with an angular velocity different from the rotational speed $\omega$, and the disk cannot move torsionally on the shaft. The dimensionless dynamic equations of the 2DOF rotor system can be obtained by reference to the study [1]. Considering effects of the internal damping and leaf springs, the dimensionless equations of motion of the rotor system are shown as follows:

$$
\left\{\begin{array}{l}
\ddot{\theta}_{x}+i_{p} \omega \dot{\theta}_{y}+c \dot{\theta}_{x}+\theta_{x}+k_{L} \theta_{x}-D_{i x}-D_{L x}=F \cos \omega t \\
\ddot{\theta}_{y}-i_{p} \omega \dot{\theta}_{x}+c \dot{\theta}_{y}+\theta_{y}+k_{L} \theta_{y}-D_{i y}-D_{L y}=F \sin \omega t
\end{array},\right.
$$

where $F=\left(1-i_{p}\right) \tau \omega^{2}, c$ is the coefficient of the external damping, and $i_{p}$ is the ratio of the principal axis of the polar moment of inertia of the disk and the diametrical moment of inertia of the disk. $D_{i x}$ and $D_{i y}$ represent the internal damping force in $x$ and $y$ directions, and $D_{L x}$ and $D_{L y}$ represent the damping force of leaf springs in $x$ and $y$ directions. $k_{L}$ is the increase of spring stiffness of the rotor due to leaf springs. They will be illustrated later in this paper.

\subsection{Internal Damping (Structural Damping)}

The pre-tightening force needs to be large enough to mount the disk on the shaft. When the deflection 
of the shaft is comparatively large, the fibres of the shaft elongate or contract with the changes of the shaft orbit, which causes the pre-tightening force to be insufficient. Thus, the static friction cannot prevent the sliding between the shaft and the disk, and the type of friction has been transformed into dry friction. The dry friction, as internal friction, will cause structural damping. In addition, a large deflection of the shaft means that there is hysteretic internal damping due to frictions in the shaft material. In order to discuss influences of the structural damping on self-excited vibrations, it is necessary to eliminate the effects of the hysteretic internal damping caused by the deflection motion, which is the reason that the 2DOF model with an inclination motion is adopted.

Here, the Coulomb friction is applied to approximate the dry friction between the shaft and the disk. The internal damping force is determined by the difference in the whirling angular velocity and rotational speed.

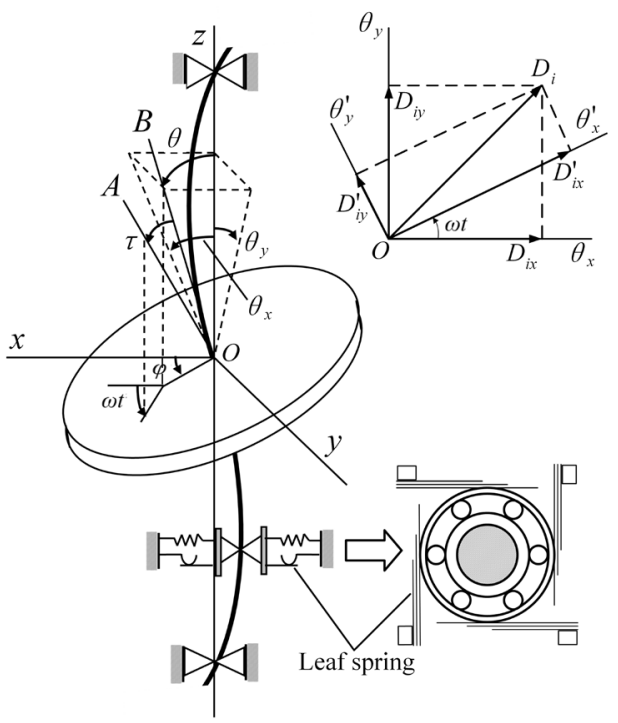

Fig. 1. Rotor model with leaf springs and coordinate system

Therefore, the internal damping force is discussed in the rotational coordinate system $O-\theta_{x}^{\prime} \theta_{y}^{\prime}$ shown in Fig. 1. For simplicity of representation, complex variables $z=\theta_{x}+i \theta_{y}$ in the coordinate system $O-\theta_{x} \theta_{y}$ and $z^{\prime}=\theta_{x}^{\prime}+i \theta_{y}^{\prime}$ in the coordinate system $O-\theta_{x}^{\prime} \theta_{y}^{\prime}$ are introduced. Based on the previous studies [1], the expression of the internal damping force in the rotational coordinate system $O-\theta_{x}^{\prime} \theta_{y}^{\prime}$ is obtained as follows:

$$
D_{i}^{\prime}=D_{i x}^{\prime}+i D_{i y}^{\prime}=-h \frac{\dot{z}^{\prime}}{\left|\dot{z}^{\prime}\right|}
$$

The expression of the internal damping force is converted to the expression in the stationary coordinate system $O-\theta_{x} \theta_{y}$ as follows:

$$
\left\{\begin{array}{l}
D_{i}=D_{i}^{\prime} e^{i \omega t}=-h \frac{\left(\dot{\theta}_{x}+\omega \theta_{y}\right)+i\left(\dot{\theta}_{y}-\omega \theta_{x}\right)}{\sqrt{\left(\dot{\theta}_{x}+\omega \theta_{y}\right)^{2}+\left(\dot{\theta}_{y}-\omega \theta_{x}\right)^{2}}} \\
D_{i x}=-h \frac{\left(\dot{\theta}_{x}+\omega \theta_{y}\right)}{\sqrt{\left(\dot{\theta}_{x}+\omega \theta_{y}\right)^{2}+\left(\dot{\theta}_{y}-\omega \theta_{x}\right)^{2}}} \\
D_{i y}=-h \frac{\left(\dot{\theta}_{y}-\omega \theta_{x}\right)}{\sqrt{\left(\dot{\theta}_{x}+\omega \theta_{y}\right)^{2}+\left(\dot{\theta}_{y}-\omega \theta_{x}\right)^{2}}}
\end{array}\right.
$$

where $h$ is a constant coefficient.

\subsection{Damping Force and Elastic Force of the Leaf Spring}

The damping force and elastic force of leaf springs work on the elastic shaft by the contact between the bearing and leaf springs shown in Fig. 1.

Under the condition with effects of the elastic force of leaf springs, the spring stiffness of the rotor system becomes larger. It is considered that the change of the spring stiffness is linear, and the increase of the restoring force of the rotor system are as follows:

$$
\left\{\begin{array}{l}
F_{L x}=-k_{L} \theta_{x} \\
F_{L y}=-k_{L} \theta_{y}
\end{array}\right.
$$

The leaf springs periodically deform with the motion of the rotor system. The dry friction of leaf springs is not negligible in the system. The restoring force has a hysteresis characteristic due to the dry friction, as shown in Fig. 2. The damping forces of leaf springs can be approximately described as follows:

$$
D_{L x}=-h_{L} \frac{\dot{\theta}_{x}}{\left|\dot{\theta}_{x}\right|}, D_{L y}=-h_{L} \frac{\dot{\theta}_{y}}{\left|\dot{\theta}_{y}\right|},
$$

where $h_{L}$ is a function of the magnitude of a relative velocity $\left|\dot{\theta}_{x}\right|$ or $\left|\dot{\theta}_{y}\right|$.

This damping force increases with the increase of the preload between leaves, and the viscous damping force increases in proportion to the velocity of the movement. Here, the dry friction is also approximated by the Coulomb friction. With the above approximation, $h_{L}$ is considered as a constant coefficient, and coulomb damping force is independent of the velocity.

\subsection{Natural Frequency Equation and Major Critical Speed}

With regard to leaf springs, the natural frequency equation of the rotor system is obtained as follows: 


$$
\left(1+h_{L}\right)+i_{p} \omega p-p^{2}=0 .
$$

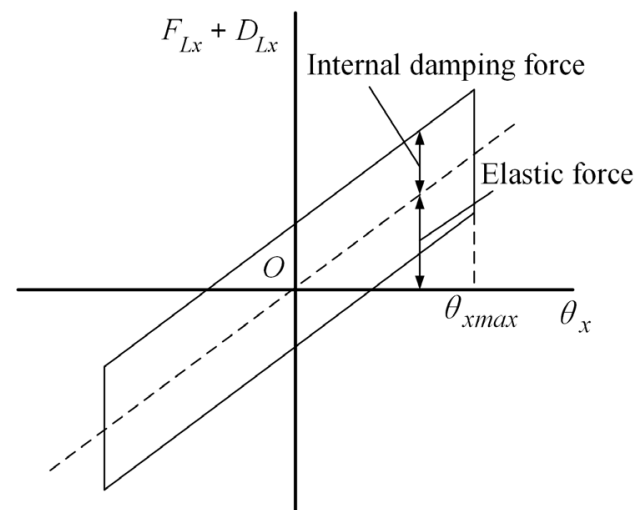

Fig. 2. Hysteretic characteristic of leaf springs

The relationship between natural frequencies and the rotational speed is obtained by solving Eq. (6), and the results are shown in Fig. 3. There are two natural frequencies $p_{f}<0$ and $p_{b}>0$ shown in Fig. 3. The $p_{f}$ is a natural frequency of a forward whirling mode and $p_{b}$ is that of a backward whirling mode. In addition, the major critical speed can be expressed as follows:

$$
\omega_{c}=\sqrt{\frac{\left(1+h_{L}\right)}{\left(1-i_{p}\right)}} .
$$

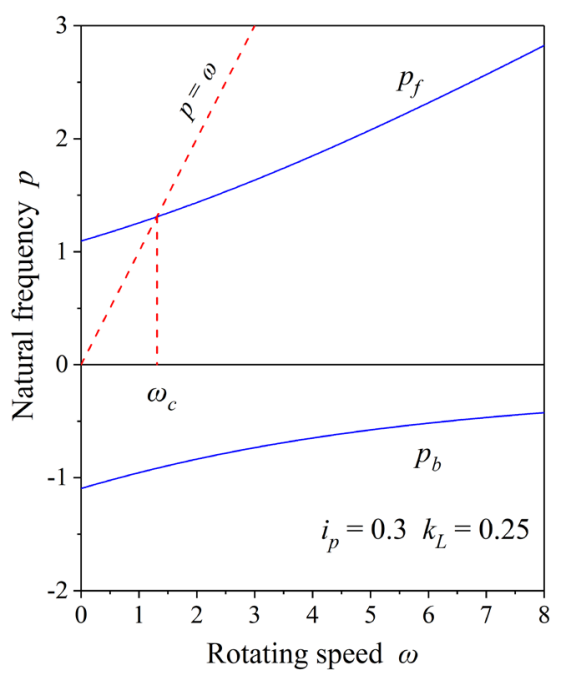

Fig. 3. Natural frequency of the 2 DOF system

\section{THEORETICAL ANALYSIS WITHOUT IMBALANCE}

In this chapter, the theoretical analysis is executed under the case without an imbalance $(\tau=0)$. Because self-excited vibrations occur above the major critical speed, the following analyses will set the case of $\omega>\omega_{c}$. In addition, because the self-excited vibration does not appear under the case of $\omega<\omega_{c}$, the solutions with zero-amplitude are stable.

\subsection{Theoretical Analysis}

When the imbalance is not considered, and the rotational speed considers the case of $\omega>\omega_{c}$, solutions for self-excited vibrations are as follows:

$$
\left\{\begin{array}{l}
\theta_{x}=R \cos \left(p_{f} t+\delta\right) \\
\theta_{y}=R \sin \left(p_{f} t+\delta\right)
\end{array} .\right.
$$

Substitute solutions Eq. (8) into the equations of motion, and represent the order of magnitude by the notation $O(\varepsilon)$ in this following. Based on the assumption that the amplitude $\mathrm{R}$ and the phase angle $\delta$ change slowly, the $\cos \left(p_{f} t+\delta\right)$ and $\sin \left(p_{f} t+\delta\right)$ will be compared with the accuracy of $O\left(e^{2}\right)$ to obtain their coefficients. follows:

The internal damping force can be expanded as

$$
\begin{gathered}
D_{i x}=-h\left(\begin{array}{c}
\frac{\dot{R} \cos \left(p_{f} t+\delta\right)}{R\left(\omega-p_{f}\right)-R \dot{\delta}+O\left(\varepsilon^{2}\right)} \\
-\frac{R\left(p_{f}+\dot{\delta}\right) \sin \left(p_{f} t+\delta\right)}{R\left(\omega-p_{f}\right)-R \dot{\delta}+O\left(\varepsilon^{2}\right)} \\
+\frac{\omega R \sin \left(p_{f} t+\delta\right)+O\left(\varepsilon^{2}\right)}{R\left(\omega-p_{f}\right)-R \dot{\delta}+O\left(\varepsilon^{2}\right)}
\end{array}\right) \\
=-h\left\{\sin \left(p_{f} t+\delta\right)+\frac{\dot{R} \cos \left(p_{f} t+\delta\right)}{R\left(\omega-p_{f}\right)}\right\}+O\left(\varepsilon^{3}\right) .
\end{gathered}
$$

The damping force of the leaf spring can be expanded as follows:

$$
\begin{aligned}
& D_{L x}=-h_{L} \frac{\dot{\theta}_{x}}{\left|\dot{\theta}_{x}\right|} \\
& =-h_{L} \frac{\cos \alpha \cos \left(p_{f} t+\delta\right)-\sin \alpha \sin \left(p_{f} t+\delta\right)}{\left|\cos \alpha \cos \left(p_{f} t+\delta\right)-\sin \alpha \sin \left(p_{f} t+\delta\right)\right|} \\
& =-h_{L} \frac{\cos \left(p_{f} t+\delta+\alpha\right)}{\left|\cos \left(p_{f} t+\delta+\alpha\right)\right|} \\
& =-\frac{4 h_{L}}{\pi} \cos \left(p_{f} t+\delta+\alpha\right)+\ldots \\
& =-\frac{4 h_{L}}{\pi}\left\{\begin{array}{c}
\frac{\dot{R} \cos \left(p_{f} t+\delta\right)}{\sqrt{\dot{R}^{2}+R^{2}\left(p_{f}+\dot{\delta}\right)^{2}}} \\
-\frac{R\left(p_{f}+\dot{\delta}\right) \sin \left(p_{f} t+\delta\right)}{\sqrt{\dot{R}^{2}+R^{2}\left(p_{f}+\dot{\delta}\right)^{2}}}
\end{array}\right\}+\ldots,
\end{aligned}
$$


where $\alpha=\arctan \frac{R(p+\dot{\delta})}{\dot{R}}$.

As a result, the equations of theoretical solutions can be obtained following:

$$
\left\{\begin{array}{l}
-R 2 p \dot{\delta}+i_{p} \omega R \dot{\delta}+c \dot{R} \\
+h \frac{\dot{R}}{R(\omega-p)}+\frac{4 h_{L}}{\pi} \frac{\dot{R}}{\sqrt{\dot{R}^{2}+R^{2}(p+\dot{\delta})^{2}}}=0 \\
-2 \dot{R} p+i_{p} \omega \dot{R}-c R(p+\dot{\delta}) \\
+h-\frac{4 h_{L}}{\pi} \frac{R(p+\dot{\delta})}{\sqrt{\dot{R}^{2}+R^{2}(p+\dot{\delta})^{2}}}=0
\end{array} .\right.
$$

\subsection{Steady-state Solution}

If the convergence of self-excited vibrations approaches a limit cycle, the steady-state solutions can be obtained as follows:

$$
R=R_{0}, \delta=\delta_{0} .
$$

The steady-state solutions can be obtained from equations given by setting derivatives of the left-hand sides of Eq. (11), which equals zero. The solution is shown as follows:

$$
R_{0}=\left(h-\frac{4 h_{L}}{\pi}\right) \frac{1}{c p_{f}} .
$$

\subsection{Stability Analysis of the Solution}

To investigate the stability of solutions, we consider small variations $\xi(t)$ and $\eta(t)$ of $O(\varepsilon)$ as follows:

$$
\left\{\begin{array}{l}
R=R_{0}+\xi \\
\delta=\delta_{0}+\eta
\end{array}\right. \text {. }
$$

Substituting Eq. (14) into Eq. (11), the small terms $O\left(\varepsilon^{3}\right)$ will be neglected, and the equations of solutions are obtained following:

$$
\left\{\begin{array}{l}
-2 p_{f} R_{0} \dot{\eta}+i_{p} \omega R_{0} \dot{\eta} \\
+\frac{h}{\left(\omega-p_{f}\right)} \frac{\dot{\xi}}{R_{0}}+\frac{4 h_{L}}{\pi} \frac{\dot{\xi}}{R_{0} p_{f}}=0 . \\
-2 \dot{\xi} p_{f}+i_{p} \omega \dot{\xi}-c \xi p_{f}=0
\end{array}\right.
$$

Assuming variations $\xi(t)$ and $\eta(t)$ to be $\xi(t)=A e^{s t}$ and $\eta(t)=B \mathrm{e}^{s t}$, and substituting them into Eq. (15), Eq. (16) can be obtained as follows:

$$
s=-\frac{c p_{f}}{\left(2 p_{f}-i_{p} \omega\right)}<0 .
$$

Obviously, the solutions are always stable because $\xi(t)$ and $\eta(t)$ are convergent.

The resonance response curves are obtained by numerical simulations and the theoretical analysis, and the results are shown in Fig. 4. The solid lines represent stable solutions. Fig. 4 shows three cases of the damping force of leaf springs, such as $h_{L}=0$ with $k_{L}=0, h_{L}=0.005$ with $k_{L}=0.25$ and $h_{L}=0.01$ with $k_{L}=0.25$. The dotted line I represents the case without leaf springs, and the dotted line II represents the case with leaf springs. The figure shows that each case of the system always has a stable solution in the wide rotational speed range and the amplitude gradually becomes smaller when the rotational speed increases. Under the conditions with leaf springs, the amplitude of self-excited vibrations significantly reduced with the increase of the parameter $h_{L}$. It is concluded that the leaf springs can effectively suppress self-excited vibrations.

Based on Eq. (13), self-excited vibrations do not occur under the case of $h_{L}>\pi h / 4$. The results of the above analyses show that leaf springs can effectively suppress self-excited vibrations in the wide rotational speed range.

\section{THEORETICAL ANALYSIS WITH IMBALANCE}

Due to the existence of internal damping terms, the multi-scale perturbation method and the harmonic balance method, it is difficult to theoretically analyse the vibration characteristics of the rotor system. In this paper, the improved shooting method is used to solve approximate solutions of the harmonic component.

\subsection{Theoretical Analysis}

Firstly, we reduce the order of Eq. (1). Putting $\Delta_{\theta_{x}}=\dot{\theta}_{x}, \Delta_{\theta_{v}}=\dot{\theta}_{y}$, Eq. (17) with four variables can be obtained as follows:

$$
\begin{aligned}
& {\left[\begin{array}{l}
\dot{\theta}_{x} \\
\dot{\Delta}_{\theta_{x}} \\
\dot{\theta}_{y} \\
\dot{\Delta}_{\theta_{y}}
\end{array}\right]=\left[\begin{array}{l}
f_{1}\left(\theta_{x}, \Delta_{\theta_{x}}, \theta_{y}, \Delta_{\theta_{y}}\right) \\
f_{2}\left(\theta_{x}, \Delta_{\theta_{x}}, \theta_{y}, \Delta_{\theta_{y}}\right) \\
f_{3}\left(\theta_{x}, \Delta_{\theta_{x}}, \theta_{y}, \Delta_{\theta_{y}}\right) \\
f_{4}\left(\theta_{x}, \Delta_{\theta_{x}}, \theta_{y}, \Delta_{\theta_{y}}\right)
\end{array}\right]} \\
& =\left[\begin{array}{l}
\Delta_{\theta_{x}} \\
F \cos \omega t-i_{p} \omega \Delta_{\theta_{y}}-c \Delta_{\theta_{x}}-\left(1+k_{L}\right) \theta_{x}+D_{i x}+D_{L x} \\
\Delta_{\theta_{y}} \\
F \sin \omega t+i_{p} \omega \Delta_{\theta_{x}}-c \Delta_{\theta_{y}}-\left(1+k_{L}\right) \theta_{y}+D_{i y}+D_{L y}
\end{array}\right],
\end{aligned}
$$


where $D_{i x}=-h \frac{\Delta_{\theta_{x}}+\omega \theta_{y}}{\sqrt{\left(\Delta_{\theta_{x}}+\omega \theta_{y}\right)^{2}+\left(\Delta_{\theta_{y}}-\omega \theta_{x}\right)^{2}} \mid}$,

$D_{i y}=-h \frac{\Delta_{\theta_{y}}-\omega \theta_{x}}{\left|\sqrt{\left(\Delta_{\theta_{x}}+\omega \theta_{y}\right)^{2}+\left(\Delta_{\theta_{y}}-\omega \theta_{x}\right)^{2}}\right|}$,

$D_{L x}=-h_{L} \frac{\Delta_{\theta_{x}}}{\left|\Delta_{\theta_{x}}\right|}$ and $D_{L y}=-h_{L} \frac{\Delta_{\theta_{y}}}{\left|\Delta_{\theta_{y}}\right|}$.

The components of the harmonic vibration are the main interest in the theoretical analyses, and it is considered that vibration components of constant terms are small. Therefore, we can assume the solutions to be as follows:

$$
\left\{\begin{array}{l}
\theta_{x}=P_{\theta x} \cos \left(\omega t+\varphi_{1}\right) \Delta_{\theta_{x}}=P_{\Delta_{\theta x}} \sin \left(\omega t+\varphi_{1}\right) \\
\theta_{y}=P_{\theta y} \sin \left(\omega t+\varphi_{1}\right) \Delta_{\theta_{y}}=P_{\Delta_{\theta_{y}}} \cos \left(\omega t+\varphi_{1}\right)
\end{array}\right.
$$

Substituting the solution Eq. (18) into Eq. (17), we make $\omega \mathrm{t}=2 n \pi$ ( $n$ represents the number of cycles in the system response) to eliminate time parameters in the system. Nonlinear equations of five variables can be obtained as follows:

$$
\begin{aligned}
& {\left[\begin{array}{l}
-P_{\theta x} \omega \sin \left(\varphi_{1}\right) \\
P_{\Delta_{\theta_{x}}} \omega \cos \left(\varphi_{1}\right) \\
P_{\theta y} \omega \cos \left(\varphi_{1}\right) \\
-P_{\Delta_{\theta_{y}}} \omega \sin \left(\varphi_{1}\right)
\end{array}\right]=} \\
& {\left[\begin{array}{l}
P_{\Delta_{\theta_{x}}} \sin \left(\varphi_{1}\right) \\
F-i_{p} \omega P_{\Delta_{\theta_{y}}} \cos \left(\omega t+\varphi_{1}\right)-c P_{\Delta_{\theta_{x}}} \sin \left(\omega t+\varphi_{1}\right) \\
-\left(1+k_{L}\right) P_{\theta x} \cos \left(\omega t+\varphi_{1}\right)+D_{i x}^{\prime}+D_{L x}^{\prime} \\
P_{y} \cos \left(\varphi_{1}\right) \\
i_{p} \omega P_{\Delta_{\theta_{x}}} \sin \left(\omega t+\varphi_{1}\right)-c P_{\Delta_{\theta_{y}}} \cos \left(\omega t+\varphi_{1}\right) \\
\quad-\left(1+k_{L}\right) P_{\theta y} \sin \left(\omega t+\varphi_{1}\right)+D_{i y}^{\prime}+D_{L y}^{\prime}
\end{array}\right] .}
\end{aligned}
$$

Therefore, the optimal numerical solution of each parameter in Eq. (19) can be solved by using the genetic algorithm.

\subsection{Stability Analysis of the Solution}

This paper applies the first Lyapunov method for the stability of approximate solutions. According to Eq. (17), the Jacobian matrix $\mathbf{A}$ can be obtained as follows:

$$
\mathbf{A}=\left[\begin{array}{llll}
\frac{\partial f_{1}}{\partial \theta_{x}} & \frac{\partial f_{1}}{\partial \Delta_{\theta_{x}}} & \frac{\partial f_{1}}{\partial \theta_{y}} & \frac{\partial f_{1}}{\partial \Delta_{\theta_{y}}} \\
\frac{\partial f_{2}}{\partial \theta_{x}} & \frac{\partial f_{2}}{\partial \Delta_{\theta_{x}}} & \frac{\partial f_{2}}{\partial \theta_{y}} & \frac{\partial f_{2}}{\partial \Delta_{\theta_{y}}} \\
\frac{\partial f_{3}}{\partial \theta_{x}} & \frac{\partial f_{3}}{\partial \Delta_{\theta_{x}}} & \frac{\partial f_{3}}{\partial \theta_{y}} & \frac{\partial f_{3}}{\partial \Delta_{\theta_{y}}} \\
\frac{\partial f_{4}}{\partial \theta_{x}} & \frac{\partial f_{4}}{\partial \Delta_{\theta_{x}}} & \frac{\partial f_{4}}{\partial \theta_{y}} & \frac{\partial f_{4}}{\partial \Delta_{\theta_{y}}}
\end{array}\right] .
$$

Substituting theoretical solutions into matrix A to obtain the coefficient matrix, the characteristic equation of the rotor system can be obtained as follows:

$$
\left[\begin{array}{cccc}
a_{11}-\lambda & a_{12} & a_{13} & a_{14} \\
a_{21} & a_{22}-\lambda & a_{23} & a_{24} \\
a_{31} & a_{32} & a_{33}-\lambda & a_{34} \\
a_{41} & a_{42} & a_{43} & a_{44}-\lambda
\end{array}\right]=0 .
$$

The stability of theoretical solutions can be investigated by judging the positive and negative of the real part of the eigenvalue $\lambda_{i}$. If all the real parts of eigenvalues are negative, the solution is stable. If there is at least one positive real part and others are negative, the solution is unsteady.

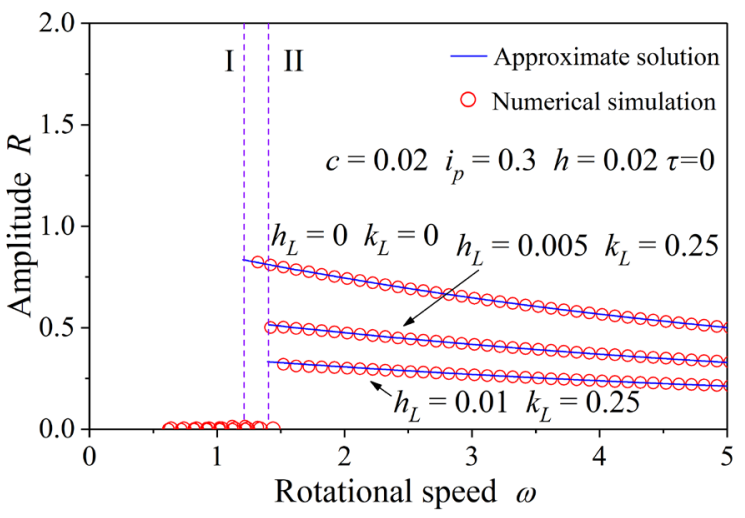

Fig. 4. Resonance responses without an imbalance

Through the above process, theoretical solutions are obtained and shown in Fig. 5. In the figure, the solid lines represent the stable solutions. The results show that the resonance curve of the harmonic vibration is a typical resonance curve of the $2 \mathrm{DOF}$ rotor, and the harmonic vibration and self-excited vibration are mutually independent. 


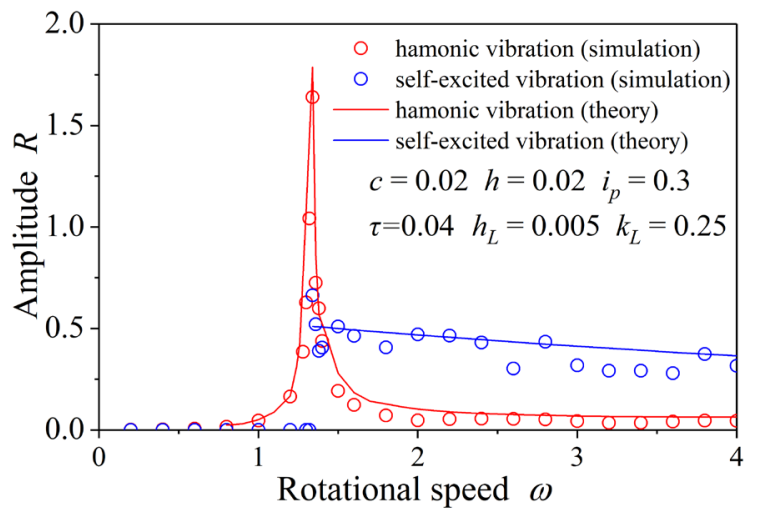

Fig. 5. Resonance responses with an imbalance

\section{NUMERICAL SIMULATION}

\subsection{Simulation without Imbalance}

Based on Eq. (1) with $\tau=0$, results of the numerical simulation without considering an Imbalance are shown in Fig. 4. The self-excited vibration occurs on the line I under the case without a leaf spring in the rotor system, and the vibration response appears on the line II under the case with leaf springs. The results of simulations consist well with results of the theoretical analysis in Chapter 3. When $h_{L}=0.005$ and $\omega=2$, the frequency components of the response are shown in Fig. 6. This frequency corresponds to the frequency $p_{f}$ shown in Fig. 3, indicating that self-excited vibrations occur in the rotor system.

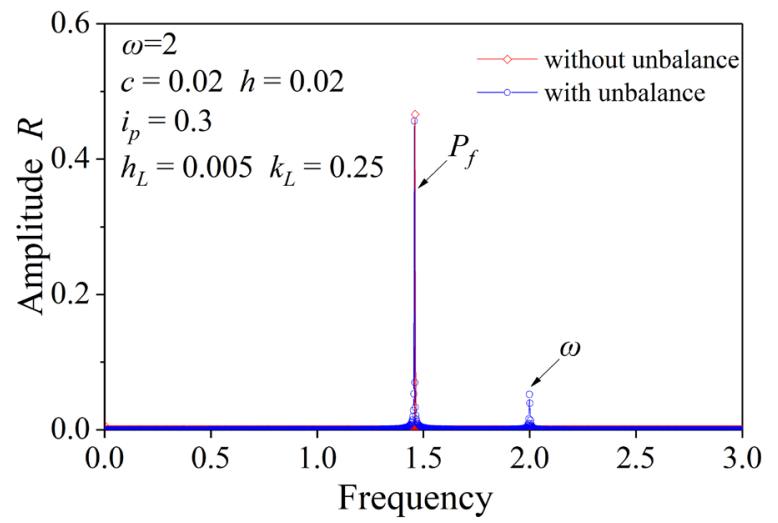

Fig. 6. Spectrum of the response

\subsection{Simulation with Imbalance}

Based on Eq. (1) with $\tau \neq 0$, numerical simulations considering an Imbalance are shown in Fig. 7. The almost-periodic motions occur above the major critical speed, and the range of the amplitude becomes smaller with the increase of the rotational speed. Fig. 6 shows frequency components of the system response, and it is found that the frequency of self-excited vibrations remain unchanged under the case with the imbalance or without the imbalance. In addition, Fig. 8 shows that beat vibrations occur in the vicinity of the major critical speed because the frequency of self-excited vibrations is close to the frequency of the harmonic vibration.

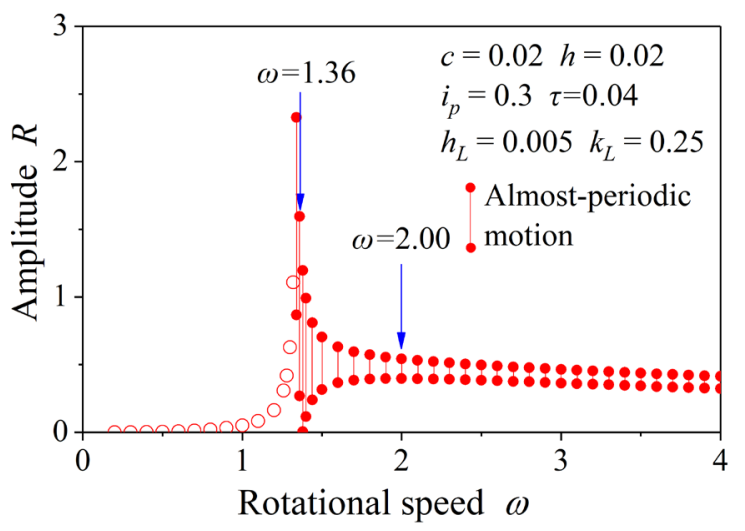

Fig. 7. Simulation with an imbalance
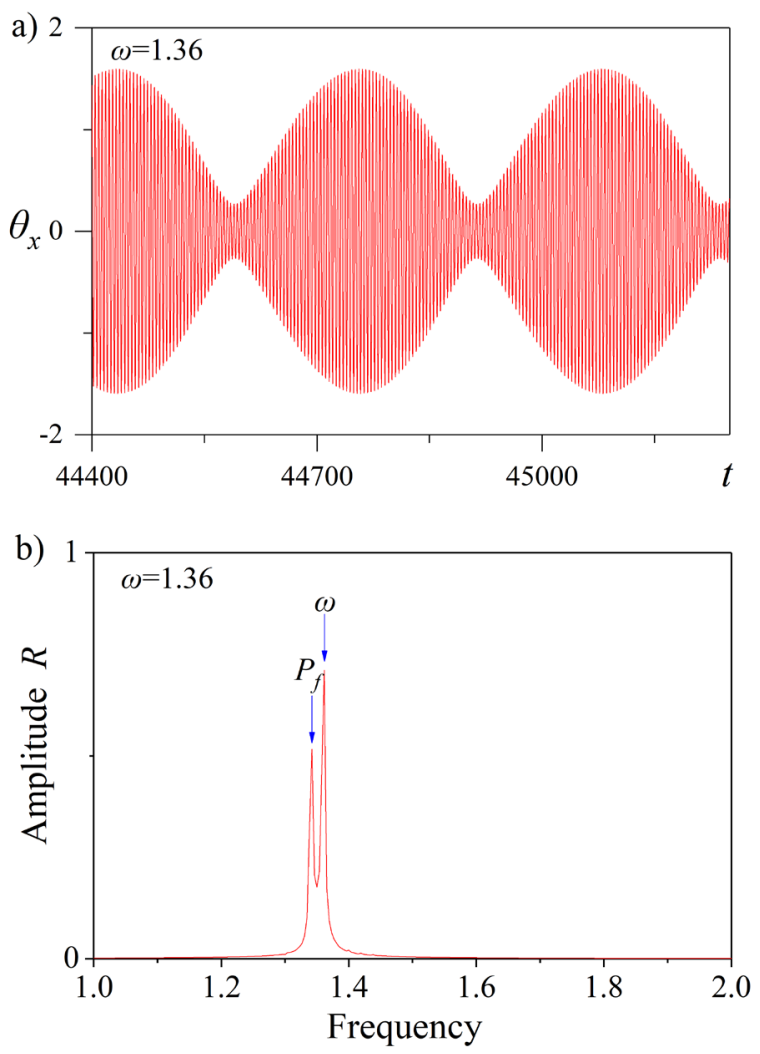

Fig. 8. Vibration response (with an imbalance); a) time history; and b) spectrum of respond 
Response curves of the harmonic vibration and self-excited vibrations obtained by simulations and theoretical analyses are shown in Fig. 5. Results show that self-excited vibrations appear above the major critical speed and the amplitude gradually decreases with the rotational speed increasing. Approximate solution curves of the harmonic vibration and selfexcited vibrations obtained in Chapter 3 are stable and consistent with the results of numerical simulations.

According to the above analyses, the harmonic vibration and self-excited vibrations are basically independent, and the almost-periodic motions occur due to the superposition of the harmonic vibration and self-excited vibrations.

\section{EXPERIMENTS}

\subsection{Experimental Device}

The structure of the experimental setup is shown in Fig. 9. The downside of the rotor system is free, which is called a cantilevered rotor system. The length of the shaft is $760 \mathrm{~mm}$, and the diameter is $12 \mathrm{~mm}$. The diameter of the disk is $260 \mathrm{~mm}$, and the thickness is $10 \mathrm{~mm}$. There is a collar to adjust the pre-tightening force on the rotor, and the internal damping force can be obtained by changing the fastening force of the collar. The ball bearing (\#6205) is installed on the downward direction from the disk by about $60 \mathrm{~mm}$. Four groups of leaf springs are provided in contact with the outer race of the bearing from four directions. Each group of leaf springs is composed of three leaves with different lengths. The dry friction can be created between leaves. In addition, two laser sensors are applied to measure radial displacements of the disk on the two orthogonal directions, and the vibration amplitude $R$ of the rotor system can be obtained.

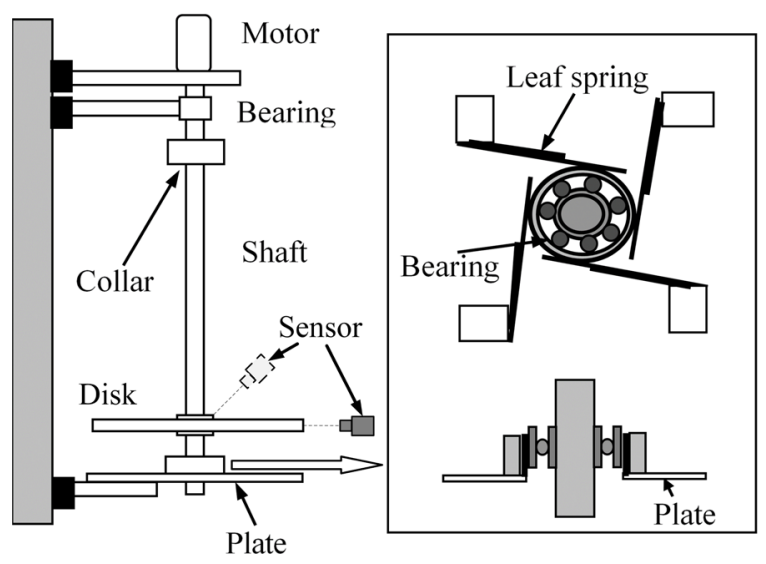

Fig. 9. Experimental setup

\subsection{Experimental Results}

The response curve of the experiment without the leaf springs is shown in Fig. 10a. The arrows indicate that the amplitude of self-excited vibrations gradually increases from the initial amplitude presented by symbol $\times$. It can be seen that self-excited vibrations occur when the rotational speed is higher than the major critical speed. Because it is very dangerous, the experiment was stopped when the amplitude is over approximately $2 \mathrm{~mm}$. The limit cycle is not validated. In addition, the time history of Point $\mathbf{A}$ is shown in Fig. 10b, and the amplitude of the vibration gradually increases from the initial amplitude.
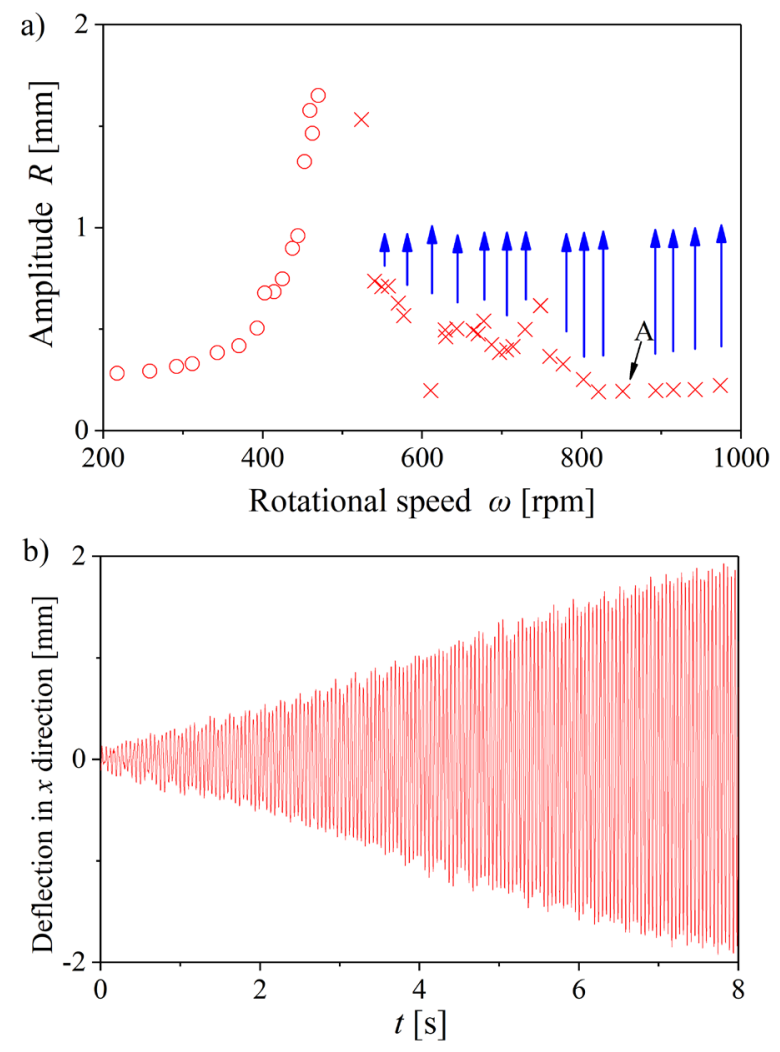

Fig. 10. Self-excited vibration (Without a leaf spring); a) resonance curve; b) time history

The response curve of the experiment with leaf springs is shown in Fig. 11. Due to the damping force of leaf springs, the resonance amplitude in the vicinity of the major critical speed becomes smaller and self-excited vibrations do not occur above the major critical speed. The measurement results are whole harmonic vibrations. For the comparison, the response curve of the experiment with leaf springs and a larger imbalance is shown in Fig. 12. Under the condition 
of Fig. 12, the major critical speed can be determined clearly, and no self-excited vibrations occur above the major critical speed. When disturbances were repeatedly applied to the rotor system, self-excited vibrations did not occur above the major critical speed.

The above experimental results show that proposed leaf springs can effectively suppress selfexcited vibrations.

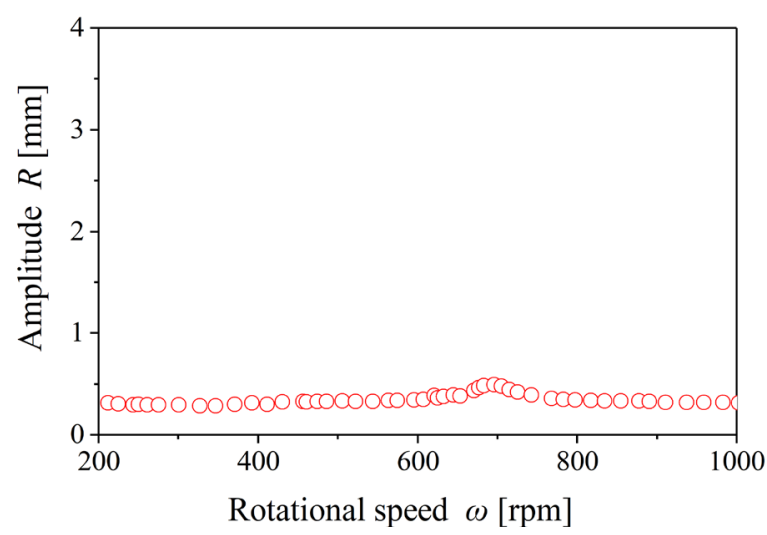

Fig. 11. Experimental result (With leaf springs)

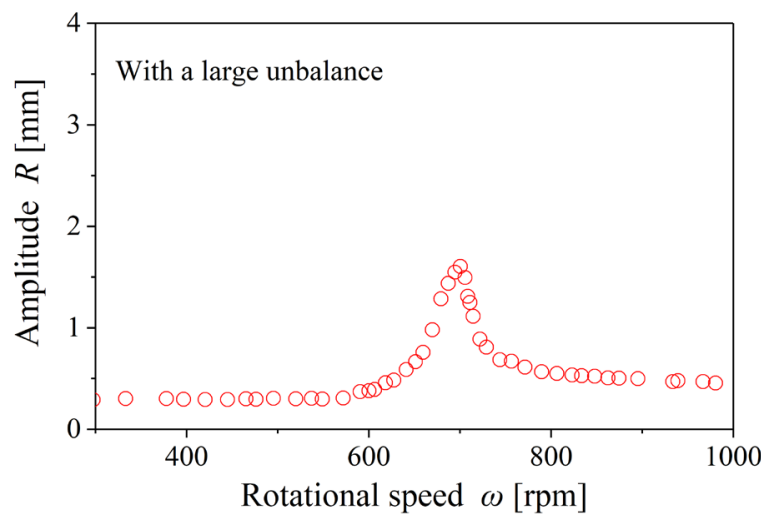

Fig. 12. Experimental result (Addition a large imbalance)

\section{CONCLUSIONS}

The self-excited vibrations caused by the structural damping occur above the major critical speed of rotating machinery. In this research, we propose a method to suppress self-excited vibrations by using leaf springs. The conclusions are as follows.

(1) The self-excited vibrations occurring above the major critical speed can be suppressed by using leaf springs.

(2) Under conditions with an imbalance, the rotor system becomes a forced autonomous system. The almost-periodic motions occur above the major critical speed due to the superposition of the harmonic vibration and self-excited vibrations.

(3) The theoretical analyses and numerical simulations can be confirmed well by experiments.

\section{ACKNOWLEDGEMENTS}

Thanks the National Key Research and Development Program of China (No.2017YFB1303304) and the Tianjin Natural Science Foundation of China (No.17JCZDJC38500) which give this paper's support.

\section{NOMENCLATURES}

$O-x y z$ rectangular coordinate system in fixed space

$\theta \quad$ inclination angle of the shaft at the position of the disk

$\theta_{x}, \theta_{y} \quad$ projections of $\theta$ onto the $x z$ - and $y z$-planes

$\theta_{x}^{\prime}, \theta_{y}^{\prime}$ parameters in rotational coordinate system

$D_{i x}^{\prime}, D_{i y}^{\prime}$ parameters in rotational coordinate system

$i_{p} \quad$ ratio of the principal axis of the polar moment of inertia of the disk and the diametrical moment of inertia of the disk

$c$ damping coefficient

$D_{i x}, D_{i y}$ internal damping forces

$D_{L x}, D_{L y}$ damping forces of the leaf spring

$K_{L} \quad$ increase of the spring stiffness

$F \quad$ amplitude of the excitation

$\varphi \quad$ initial phase angle of the excitation

$t \quad$ time

$\omega \quad$ rotational speed, [rpm]

$\omega_{c} \quad$ major critical speed, [rpm]

$\tau \quad$ inclined angle of the rotor (imbalance)

$h, h_{L} \quad$ constant coefficients of the internal damping force

$p, p_{f}, p_{b}$ natural frequencies of the rotor system

$R \quad$ amplitude of vibrations of the rotor system, [mm]

$P_{\theta x}, P_{\theta y}$ amplitude of vibrations in $x$ and $y$ directions

$P_{x}, P_{y}$ first derivative term of amplitude of vibrations in $x$ and $y$ directions

$\delta, \varphi_{1} \quad$ phase angle of vibrations of the rotor system

$O(\varepsilon) \quad$ orders in magnitude of parameters

$\xi, \eta \quad$ small variations of $O(\varepsilon)$

$s \quad$ variation in the complex field

$\lambda$ eigenvalue of the characteristic equation of the rotor system 


\section{REFERENCES}

[1] Yamamoto, T., Ishida, Y. (2012). Linear and Nonlinear Rotordynamics: A Modern Treatment with Applications. Wiley, New York.

[2] De Queiroz, M. (2013). An active hydrodynamic bearing for controlling self-excited vibrations: theory and simulation. Journal of Vibration and Control, vol. 19, no. 14, p. 22112222, D0I:10.1177/1077546312458945.

[3] Bonello, P., Pham, H.M. (2014). The efficient computation of the nonlinear dynamic response of a foil-air bearing rotor system. Journal of Sound and Vibration, vol. 333, no. 15, p. 3459-3478, D0I:10.1016/j.jsv.2014.03.001.

[4] Boyaci, A., Lu, D.X., Schweizer, B. (2015). Stability and bifurcation phenomena of Laval/Jeffcott rotors in semifloating ring bearings. Nonlinear Dynamics, vol. 79, no. 2, p. 1535-1561, D0l:10.1007/s11071-014-1759-5.

[5] Bykov, V.G., Tovstik, P.E. (2018). Synchronous whirling and self-oscillations of a statically unbalanced rotor in limited excitation. Mechanics of Solids, vol. 53, p. 60-70, DOI:10.3103/S0025654418050047.

[6] Ishida, Y., Yamamoto, T. (1993). Forced oscillations of a rotating shaft with nonlinear spring characteristics and internal damping (1/2 order subharmonic oscillation and entrainment). Nonlinear Dynamics, vol. 4, no. 5, p. 413-431, DOI:10.1007/BF00053689.

[7] Coudeyras, N., Sinou, J.J., Nacivet, S. (2009). A new treatment for predicting the self-excited vibrations of nonlinear systems with frictional interfaces: The constrained harmonic balance method, with application to disc brake squeal. Journal of Sound and Vibration, vol. 319, no. 3-5, p. 1175-1199, DOI:10.1016/j.jsv.2008.06.050.

[8] Han, D.J., Tang, C.L., Hao, L., Yang, J.F. (2016). Experimental studies on the effects of bearing supply gas pressure on the response of a permanent magnet disk-type motor rotor. Journal of Mechanical Science and Technology, vol. 30, no. 11, p. 4887-4892, D0I:10.1007/s12206-016-1008-6.
[9] Vlajic, N., Champneys, A.R., Balachandran, B. (2017). Nonlinear dynamics of a Jeffcott rotor with torsional deformations and rotor-stator contact. International Journal of Non-linear Mechanics, vol. 92, p. 102-110, D0l:10.1016/j. ijnonlinmec.2017.02.002.

[10] Hua, C.L., Cao, G.H., Rao, Z.S., Ta, N., Zhu, Z.C. (2017). Coupled bending and torsional vibration of a rotor system with nonlinear friction. Journal of Mechanical Science and Technology, vol. 31, no. 6, p. 2679-2689, Dol:10.1007/ s12206-017-0511-8.

[11] Nishimura, A., Inoue, T., Watanabe, Y. (2018). Nonlinear analysis and characteristic variation of self-excited vibration in the vertical rotor system due to the flexible support of the journal bearing. Journal of Vibration and Acoustics, vol. 140, no. 1, Dol:10.1115/1.4037520.

[12] Tadokoro, C., Nagamine, T., Nakano, K. (2018). Stabilizing effect arising from parallel misalignment in circular sliding contact. Tribology International, vol. 120, p. 16-22, DOI:10.1016/j.triboint.2017.12.003.

[13] Chouchane, M., Amamou, A. (2011). Bifurcation of limit cycles in fluid film bearings. International Journal of Non-linear Mechanics, vol. 46, no. 9, p. 1258-1264, D0l:10.1016/j. ijnonlinmec.2011.06.005.

[14] Peletan, L., Baguet, S., Torkhani, M., Jacquet-Richardet, G. (2014). Quasi-periodic harmonic balance method for rubbing self-induced vibrations in rotor-stator dynamics. Nonlinear Dynamics, vol. 78, no. 4, p. 2501-2515, D0l:10.1007/s11071014-1606-8.

[15] Kligerman, Y., Gottlieb, O., Darlow, M.S. (1998). Nonlinear vibration of a rotating system with an electromagnetic damper and a cubic restoring force. Journal of Vibration and Control, vol. 4, no. 2, p. 131-144, DOl:10.1177/107754639800400203.

[16] Inoue, T., Ishida, Y., Niimi, H. (2012). Vibration analysis of a self-excited vibration in a rotor system caused by a ball balancer. Journal of Vibration and Acoustics, vol. 134, no. 2, DOI:10.1115/1.4005141. 